\title{
ERROR ANALYSIS OF CHINESE WORD ORDER OF INDONESIAN STUDENTS 印尼学生汉语状语语序常见偏误分析
}

\author{
Yetty Go \\ Chinese Department, Faculty of Humaniora, BinusUniverstiy \\ Jln. Kemanggisan Ilir III No.45, Kemanggisan - Palmerah, Jakarta Barat 11480 \\ yetty@binus.edu
}

\begin{abstract}
This study used comparative analysis to understand the similarities and differences of basic structure adverbial word order between Chinese language and Bahasa Indonesia. This study also analyzed Indonesian student's common errors in learning adverbial word order, and the result found that student's common error are adverbial clause of time, place, and preposition as adverbial word order. This research suggests teachers must emphasize the differences in the basic word order between Chinese language and bahasa Indonesia language first, then teach the differences of adverbial word. Teachers shoud encourage students to practice listening, speaking, reading, and writing skills, so they can overcome the differences between Chineseand Indonesia language, and reduce the errors in learning.
\end{abstract}

Keyword: Chinese language, bahasa Indonesia, adverbial, comparative, errors

\section{摘要}

本研究是透过针对汉语与印尼语状语语序的本体结构进行对比分析来了解两语言间的状语语序之异 同。本研究也针对印尼学生在学习中常见的偏误进行分析, 发现了学生的偏误: 表示时间状语的错序、 表示处所状语的错序以及介词当状语的错序。本研究建议教师在教学上, 要先强调汉印的基本语序上的 差异才强调两者语言的状语语序上的差异。教师也应该多鼓励学生多练习在听说读写语言技能, 这样学 生才能克服学习汉印状语语序的差别, 并减少学生在学习上的偏误。

关键词：汉语、印尼语、状语语序、对比、偏误 


\section{前言}

目前, 中国是世界上经济发展最快的国家之一。汉语已经成为联合国的官方语言之一。现 在在印尼, 汉语扮演很大的角色, 因为目前在印尼有很多中资台资企业, 所以汉语成为必须掌 握的语言之一。因此, 老老少少都开始学习汉语, 不只是印尼华侨, 连印尼原住民也开始学习 了。

在句子中, 状语是谓语部分的修饰成分, 主要是用来修饰动词或形容词, 是句子谓语部分 的重要组成成分（刘月华，2001）。状语是汉语句法的重要成分之一, 由于其直接对句子核心 部分或整个句子进行修饰描写。因此, 状语的表述直接影响到对整个句意的理解（洪铭隆, 2011）。

汉语与印尼语状语位置的差别使印尼学生很难以厘清状语位置的先后顺序, 在表达时常常 发生偏误。根据洪铭隆（2011）在《印尼留学生汉语词序习得偏误研究一基于“HSK 动态作文 语料库”》中得知, 状语位置的偏误在印尼学生学习汉语的过程中出现的比例非常高, 在分析 当中发现 280 例词序错句中达到 127 例, 占据 45.4\%。陈延河（2002）在《印尼语、汉语语序 对比及印尼学生汉语学习中常见语序偏误分析》中得知, 汉语有而印尼语没有的句法特殊成为 印尼学生学习汉语的难点, 这应该值得去探讨的问题。

本研究透过针对汉语与印尼语状语语序的本体结构进行对比分析来了解两者语言的状语语 序之异同, 亦针对印尼学生在学习中常见的偏误进行分析, 并针对印尼学生提供状语语序的教 学建议。

\section{研究方法}

本研究先针对印尼语与汉语状语语序的本体结构进行对比分析来了解两者语言的状语语序 之异同。本研究的主要来源是从印尼学生的写作语料库。语料库里总共有 22 篇学生写的作文, 学生是大学三年级, 他们的程度是中级程度。从语料库里, 笔者想探讨印尼学生在作文里常见 的偏误有哪些, 并以后可以为印尼学生提供教学建议, 希望可以协助学生减少使用上所产生的 偏误。

\section{內容}

\section{印尼语与汉语的状语语序之对比}

卢福波（2005）指出, 状语是汉语的修饰语其中之一。状语的定义内含, 笔者参考了刘月 华《实用现代华语文语法》、卢福波《对外华语文教学实用语法》、孙德金《华语文语法教 程》, 三本语法著作得知, 状语是谓语部分的修饰成分, 主要是用来修饰动词或形容词, 是句 子谓语部分的重要组成成分。

根据卢福波（2005），状语的位置一般出现在被修饰的中心语前面。状语的位置分为两种, 第一种是位于主语前的数量比较少; 第二种是位于主语后的数量比较多。而印尼语的状语位置 没有严格的规定, 它的语序比汉语自由、灵活得多（张琼郁, 2012）。下列是印尼语与汉语的 状语语序对比: 
（一）表示时间的状语，其位置是不固定的有的放在句首，也有的放在句尾。例如:

1. Malam ini ayah membawa kami makan malam ke restoran.

今晚爸爸带我们吃晚饭去餐厅

今晚爸爸带我们去餐厅吃晚饭。

2. Mereka pergi menonton film kemarin malam.

他们去看电影昨晚

他们昨晚去看电影。

（二）表示处所的状语，包括其空间的概念一般放在谓语的后面。例如:

3. Mereka sekeluarga tinggal di ibukota.

他们一家人住在首都

他们一家人住在首都。

4. Rumah dia tidak jauh dari sini.

家他不远从这里

他的家从这里不远。

（三）表示情态的状语一般都放在谓语前面也可以放在谓语后面。例如:

5. Perlahan-lahan kami masuk.

慢慢我们进去

我们慢慢地进去。

6. Ia berdiri diam-diam.

他站着安静

他安静地站着。

（四）程度副词 sangat“很”作状语时, 是放在形容词前面。例如:

7. Baju ini sangat murah.

衣服这很便宜

这件衣服很便宜。

（五）表示否定和肯定的状语。表示否定时一般放在谓语的前面，若表示肯定时有的放在谓 语前面，有的放在谓语后面。例如:

8. Kamu jangan tidur malam-malam.

你别睡觉很晚

你别很晚睡觉。

9. Musuh pasti dihancur-leburkan oleh rakyat.

敌人一定消灭掉被人民

敌人一定被人民消灭掉。

从上述的例子对比得知, 印尼语的状语位置大部分都可以放在谓语后面与汉语的状语位置 不同, 如印尼语表示时间与肯定的状语位置都可以放在谓语的前后, 而汉语的状语位置一般都 放在谓语前面。因为印尼语的状语位置没有很严格的规定, 其的语序比汉语自由、灵活得多, 因为这样的因素使印尼学生在学习汉语状语方面难免出现状语序的偏误。 


\section{印尼学生在状语语序中常见的偏误}

其实印尼学生在学习汉语的过程中出现的偏误是有很多种。从印尼学生产生的语序偏误来 看是有不少例子明显是因为受到母语干扰的因素, 即负迁移造成的。同样的语序的偏误比较极 少, 若是不一样又比较复杂的语序, 偏误就较多。

洪铭隆(2011)在《印尼留学生华语文词序习得偏误研究一基于“HSK 动态作文语料库”》中 得知, 状语位置的偏误在印尼留学生学习汉语的过程中出现的比例非常高, 在分析当中发现 280 例词序错句中达到 127 例，占据 45.4\%。

本研究基于印尼大学生的写作作文为语料库。语料库里总共有 22 篇三年级大学生写的作 文。印尼是母语非汉语的国家, 所以学生在学习的过程中因为母语的干扰难免会产生偏误。下 列是印尼学生在状语语序方面常见偏误:

（一）表示时间状语的错序

(1) *七点半早上, 我坐车出发。

(早上七点半, 我坐车出发。)

(2) * 那天七点早上, 我很早就起床了。

(那天早上七点, 我很早就起床了。)

例(1)和(2)正确的表达应该是「早上七点半」和「早上七点」, 这两个句子的偏误是因为 汉语文与印尼语的时间表达顺序是相反的。以上的错序主要是因为母语的干扰导致的, 虽然这 两个偏误是常产生在初级阶段, 但是也还会在中级阶段出现这种偏误。

（二）表示处所状语的错序

(3) *我的朋友和我遇到了他在商场。 (我的朋友和我在商场遇到了他。)

(4) *我很少见到他在大学。 (我很少在大学见到他。)

(5)*因为我的大学很大, 所以很难遇到他在大学。 (因为我的大学很大, 所以很难在大学遇到他。)

(6) * * 我跟他学习在一个大学。 (我跟他在一个大学学习。)

(7) *晚上我常常看他睡觉在外面的餐厅。 (晚上我常常看他在餐厅的外面睡觉。)

例(3)至(6)都把地点放在句子后面, 这是因为「在商场」、「在大学」在印尼语都习惯放 在后置。例(7)应该「在餐厅的外面」, 印尼语「方位词」的位置一般是在名词前面。这三个句 子的错序都是有母语的影响而产生的偏误。

（三）介词词组当状语的错序

（8）*佩妮已经住在巴黎跟她爸爸。

(佩妮跟她爸爸已经住在巴黎十年多了。)

(9) * 你们好! 很久没有见面跟你们了。 (你们好! 很久没有跟你们见面了。) 
以上的例子是有关介词结构当状语时，印尼语的介词多为放在谓语前，也可以放在谓语后。 但是因为口语往往会放在谓语后面, 所以学生造句时会把它写在句尾, 如例(8) 和(9)。印尼语 的介词结构当状语语序比汉语文的较灵活，所以难免出现上面例子的错序，这是负迁移的结果。 例(8)除了介词词组位置不对，「十年多了」是时间补语应该放在谓语后面。

\section{针对印尼学生状语语序教学建议}

分析了印尼学生习得汉语状语语序的偏误情况之后, 并探讨了对造成这些偏误的原因。下 列是针对印尼学生造成的偏误提出一些教学建议, 希望能够给印尼的汉语教师在教学上的一些 启发。

\section{强调汉印的基本语序上的差异}

为了克服母语对学习目的语的干扰，笔者建议教师首先要强调汉印基本语序上的差异，如 下列:

\section{1. 具有相同点的基本语序}

(1) 基本语序「SVO」的句子: Saya suka Taiwan. (我喜欢台湾)

(2) 汉语的「介系词」“在+处所”: Ibu memasak di dapur. (妈妈在厨房做饭)

(3) 汉语的「形容词片语」“很+形容词”: Guru sangat cantik. (老师很漂亮)

\section{2. 不相同点的基本语序}

(4) 汉印「了」与「telah/sudah」在句子的位置, 汉语里表示活动完成的「了」在句子的 位置是放在句尾, 如: “我吃饭了”, 而印尼语「telah/sudah」的位置是放在主谓语之 间，如: “Saya telah makan nasi”。

(5) 汉印「过」与「pernah」在句子的位置, 汉语「过」在句子的位置是放在动词后面, 如：“我去过台湾”，而印尼语「pernah」的位置是放在主谓语之间。

(6) 汉印「的」与「kepunyaan」的位置, 汉语里表示所有者, 会把所有者放在所有物的前, 前面的定语修饰后面的中心语，定语和中心语之间会加「的」，如：“我的爸爸”，“弟 弟的书包”。而印尼语把所有者放在所有物的后面，后面的定语修饰前面的中心语。

上述的基本语序是印尼学生常因为母语的干扰而产生偏误, 特别是初级阶段的学生。学生 若能够掌握华印的基本语序上的相似之处和不同所在，可减少学习中的负迁移作用。

\section{强调汉印的状语语序上的差异}

印尼语的状语位置比较灵活, 而汉语比较固定, 这样的情况印尼学生难免发生一些偏误。 笔者建议教师先教表示时间与表示处所的状语。汉语表示时间的状语时的位置可以放在主语前 面或者主语后面, 如: “明天我去台北”, “爸爸晚上八点看电视”, 而在印尼语, 其位置是可放 在句首与句尾, 如: “Saya pergi ke Taipei esok hari”, “Besok saya pergi ke Taipei”。汉语的表示 处所的状语在句子里的位置是放在谓语前面, 如: “弟弟在房间读书”。若谓语前有加数量词可 以放在谓语后, 如: “他放一本书在桌上”。而印尼语的话, 无论谓语前有没有加数量词, 若谓 语前有加数量词会放在谓语后。汉语介词词组当状语「跟+名词」的位置是放在主语谓语前, 如: “我跟安妮去书店”, 而印尼语是放在谓语后面, 如: “Saya pergi ke toko buku dengan Ani”。 


\section{强化印尼学生的汉语感}

周健(2003)指出, 从培养学生语感的目的开始, 汉语课堂教学就应当让学生多读、多听、 多说、多写、多开展形式多样的课堂交际活动。解决学生的语序方面, 最重要是培养学生的汉 语感。教师在课堂上要多鼓励学生做很多练习, 这样学生才能克服汉印的差异。

\section{结语}

本研究通过对比汉印的状语语序得知, 汉语的状语语序比较固定, 印尼语的状语语序比较 灵活。由于这样的因素, 学生在学习汉语时难免产生偏误。本研究基于印尼三年级大学生的 22 篇作文为语料来源, 笔者通过学生的作文考试来分析印尼学生常见的汉语状语语序的偏误。 分析后，笔者发现印尼学生的错序偏误包括表示时间的状语、表示处所的状语和介词当状语。 笔者建议教师在教学上要先强调汉印的基本语序上的差异才强调汉印的状语语序上的差异。教 师也应该多鼓励学生在听说读写语言技能多练习，这样学生才能克服汉语的学习困难。

\section{参考文献}

刘月华.（2001）.实用现代华语文语法。北京：商务印书馆。

卢福波.（2005）.对外华语文教学实用语法北京：北京语言大学出版社。

张琼郁.（2012）.现代印尼语语法北京：外语教学与研究出版社。

孙德金.（2003）.华语文语法教程北京：北京语言大学出版社。

房玉清.（2008）.实用华语文语法北京：北京语言大学出版社。

陳延河.（2002）.印尼語、華語文詞序對比及印尼學生華語文學習中常見詞序偏誤分析。《暨 南人學華文學院學》，1，9-18。

洪銘隆(2011)。印尼留學生華語文詞序習得偏誤研究一基於 “HSK 動態作文語料庫”。上海師 範大學語言學及應用語言學碩士論文。

Aziz., A., Adlan, \& Minanurahman. Pedoman Umum Tata Bahasa Indonesia. Jakarta: Bee Media Indonesia 